\title{
Erratum to: Comment on: Gleicher N et al.,2016. Reprod biol endocrinol Sep 5;14(1):54
}

Ashley W. Tiegs ${ }^{1 *}$, James A. Grifo², Santiago Munné3 ${ }^{3}$ David H. McCulloh and Brooke Hodes-Wertz ${ }^{2}$

\section{Erratum}

The original article [1], was published with the incorrect title "Response to comment on: Gleicher N et al., 2016. Reprod biol endocrinol Sep 5;14(1):54". The title of this article should read "Comment on: Gleicher $\mathrm{N}$ et al., 2016. Reprod biol endocrinol Sep 5;14(1):54" and has now also been corrected online.

\section{Author details}

${ }^{1}$ Department of Obstetrics and Gynecology, New York University School of Medicine, 550 First Avenue, NBV 9E2, New York 10016, NY, USA. ${ }^{2}$ New York University Fertility Center, New York University School of Medicine, 660 First Avenue, 5th floor, New York 10016, NY, USA. ${ }^{3}$ Reprogenetics, New York, USA.

Received: 5 April 2017 Accepted: 5 April 2017

Published online: 10 April 2017

\section{Reference}

1. Tiegs AW, Grifo JA, Munné S, McCulloh DH, Hodes-Wertz B. Response to comment on: Gleicher N et al,2016. Reprod biol endocrinol Sep 5;14(1):54. Reprod Biol Endocrinol. 2017;17(15):24.

\footnotetext{
* Correspondence: Ashley.Wood@nyumc.org

'Department of Obstetrics and Gynecology, New York University School of Medicine, 550 First Avenue, NBV 9E2, New York 10016, NY, USA
} 